\title{
Existence Results for a Fully Fourth-Order Boundary Value Problem
}

\author{
Yongxiang Li and Qiuyan Liang \\ Department of Mathematics, Northwest Normal University, Lanzhou 730070, China \\ Correspondence should be addressed to Yongxiang Li; liyxnwnu@163.com
}

Received 21 December 2012; Accepted 27 June 2013

Academic Editor: To Ma

Copyright (C) 2013 Y. Li and Q. Liang. This is an open access article distributed under the Creative Commons Attribution License, which permits unrestricted use, distribution, and reproduction in any medium, provided the original work is properly cited.

We discuss the existence of solution for the fully fourth-order boundary value problem $u^{(4)}=f\left(t, u, u^{\prime}, u^{\prime \prime}, u^{\prime \prime \prime}\right), 0 \leq t \leq 1, u(0)=$ $u(1)=u^{\prime \prime}(0)=u^{\prime \prime}(1)=0$. A growth condition on $f$ guaranteeing the existence of solution is presented. The discussion is based on the Fourier analysis method and Leray-Schauder fixed point theorem.

\section{Introduction and Main Results}

In this paper we deal with the existence of solution for the fully fourth-order ordinary differential equation boundary value problem (BVP)

$$
\begin{gathered}
u^{(4)}(t)=f\left(t, u(t), u^{\prime}(t), u^{\prime \prime}(t), u^{\prime \prime \prime}(t)\right), \quad 0 \leq t \leq 1, \\
u(0)=u(1)=u^{\prime \prime}(0)=u^{\prime \prime}(1)=0,
\end{gathered}
$$

where $f:[0,1] \times \mathbb{R}^{4} \rightarrow \mathbb{R}$ is continuous. This problem models deformations of an elastic beam whose two ends are simply supported in equilibrium state, and its research has important significance in mechanics.

For the special case of BVP(1) that $f$ does not contain derivative terms $u^{\prime}$ and $u^{\prime \prime \prime}$, namely, simply fourth-order boundary value problem

$$
\begin{gathered}
u^{(4)}(t)=f\left(t, u(t), u^{\prime \prime}(t)\right), \quad 0 \leq t \leq 1, \\
u(0)=u(1)=u^{\prime \prime}(0)=u^{\prime \prime}(1)=0,
\end{gathered}
$$

the existence of solution has been studied by many authors; see [1-8]. In [1], Aftabizadeh showed the existence of a solution to PBV(2) under the restriction that $f$ is a bounded function. In [2, Theorem 1], Yang extended Aftabizadeh's result and showed the existence for BVP(2) under the growth condition of the form

$$
|f(t, u, v)| \leq a|u|+b|v|+c
$$

where $a, b$, and $c$ are positive constants such that

$$
\frac{a}{\pi^{4}}+\frac{b}{\pi^{2}}<1
$$

In [3], under a more general linear growth condition of two-parameter nonresonance, del Pino and Manásevich also discussed the existence of BVP(2) and the result of Yang was further extended. For more results involving two-parameter nonresonance condition see [4, 7]. All these works are based on Leray-Schauder degree theory. In $[5,6]$, the upper and lower solutions method is applied to discuss the existence of BVP(2). Recently, in [8] the fixed point index theory in cones is employed to $\mathrm{BVP}(2)$ and some existence results of positive are obtained, where $f$ may be super-linear growth.

For the more simple case of BVP(1) that $f$ does not contain any derivative terms, the following fourth-order boundary value problem

$$
\begin{gathered}
u^{(4)}(t)=f(t, u(t)), \quad 0 \leq t \leq 1, \\
u(0)=u(1)=u^{\prime \prime}(0)=u^{\prime \prime}(1)=0,
\end{gathered}
$$

has been studied by more researchers, and various theorems and methods of nonlinear analysis have been applied; see [913] and reference therein.

However, few researchers consider the fully fourth-order boundary value problem BVP(1). The purpose of this paper is to discuss the existence of solution of BVP(1). We will extend the Yang's result previously mentioned from BVP(2) to the general BVP(1). Our results are as follows. 
Theorem 1. Assume that $f \in C\left([0,1] \times \mathbb{R}^{4}, \mathbb{R}\right)$ and it satisfies the growth condition

$$
\begin{aligned}
& \left|f\left(t, x_{0}, x_{1}, x_{2}, x_{3}\right)\right| \\
& \quad \leq c_{0}\left|x_{0}\right|+c_{1}\left|x_{1}\right|+c_{2}\left|x_{2}\right|+c_{3}\left|x_{3}\right|+M
\end{aligned}
$$

for all $t \in[0,1]$ and $\left(x_{0}, x_{1}, x_{2}, x_{3}\right) \in \mathbb{R}^{4}$, where $c_{0}, c_{1}, c_{2}, c_{3} \geq 0$ and $M>0$ are constants and $c_{0}, c_{1}, c_{2}, c_{3}$ satisfy the restriction

$$
\frac{c_{0}}{\pi^{4}}+\frac{c_{1}}{\pi^{3}}+\frac{c_{2}}{\pi^{2}}+\frac{c_{3}}{\pi}<1 .
$$

Then the BVP(1) possesses at least one solution.

Theorem 1 is a directly extension of Yang's result previously mentioned. In Theorem 1, the condition (7) is optimal. If the condition (7) does not hold, the existence of solution of BVP(1) cannot be guaranteed. Strengthening the condition (6) of Theorem 1, we can obtain the following uniqueness result.

Theorem 2. Assume that $f \in C\left([0,1] \times \mathbb{R}^{4}, \mathbb{R}\right)$ and it satisfies the Lipschitz-type condition

$$
\left|f\left(t, x_{0}, x_{1}, x_{2}, x_{3}\right)-f\left(t, y_{0}, y_{1}, y_{2}, y_{3}\right)\right| \leq \sum_{i=0}^{3} c_{i}\left|x_{i}-y_{i}\right|
$$

for any $\left(t, x_{0}, x_{1}, x_{2}, x_{3}\right)$ and $\left(t, y_{0}, y_{1}, y_{2}, y_{3}\right) \in[0,1] \times \mathbb{R}^{4}$, where $c_{0}, c_{1}, c_{2}, c_{3} \geq 0$ are constants and satisfy (7). Then $B V P(1)$ has a unique solution.

If the partial derivatives $f_{x_{0}}, f_{x_{1}}, f_{x_{2}}$, and $f_{x_{3}}$ exist, then from Theorem 2 and the theorem of differential mean value, we have the following.

Corollary 3. Let $f \in C\left([0,1] \times \mathbb{R}^{4}, \mathbb{R}\right)$ and the partial derivatives $f_{x_{0}}, f_{x_{1}}, f_{x_{2}}$, and $f_{x_{3}}$ exist. If there exist positive constants $c_{0}, c_{1}, c_{2}, c_{3}$ such that

$$
\left|f_{x_{i}}\left(t, x_{0}, x_{1}, x_{2}, x_{3}\right)\right| \leq c_{i}, \quad i=0,1,2,3,
$$

and the constants $c_{0}, c_{1}, c_{2}, c_{3}$ satisfy (7), then BVP(1) has one unique solution.

The proofs of Theorems 1 and 2 are based on the Fourier analysis method and Leray-Schauder fixed point theorem, which will be given in Section 2 .

\section{Proof of the Main Results}

Let $I=[0,1]$ and $H=L^{2}(I)$ be the usual Hilbert space with the interior product $(u, v)=\int_{0}^{1} u(t) v(t) d t$ and the norm $\|u\|_{2}=\left(\int_{0}^{1}|u(t)|^{2} d t\right)^{1 / 2}$. For $m \in \mathbb{N}$, let $W^{m, 2}(I)$ be the usual Sobolev space with the norm $\|u\|_{m, 2}=\sqrt{\sum_{i=0}^{m}\left\|u^{(i)}\right\|_{2}^{2}} \cdot u \in$ $W^{m, 2}(I)$ means that $u \in C^{m-1}(I), u^{(m-1)}(t)$ is absolutely continuous on $I$ and $u^{(m)} \in L^{2}(I)$.
Given $h \in L^{2}(I)$, we consider the linear fourth-order boundary value problem (LBVP)

$$
\begin{gathered}
u^{(4)}(t)=h(t), \quad t \in I, \\
u(0)=u(1)=u^{\prime \prime}(0)=u^{\prime \prime}(1)=0 .
\end{gathered}
$$

Let $G(t, s)$ be the Green's function to the second-order linear boundary value problem

$$
-u^{\prime \prime}=0, \quad u(0)=u(1)=0,
$$

which is explicitly expressed by

$$
G(t, s)= \begin{cases}t(1-s), & 0 \leq t \leq s \leq 1 \\ s(1-t), & 0 \leq s \leq t \leq 1\end{cases}
$$

For every given $h \in L^{2}(I)$, it is easy to verify that the $\operatorname{LBVP}(10)$ has a unique solution $u \in W^{4,2}(I)$ in Carathéodory sense, which is given by

$$
u(t)=\iint_{0}^{1} G(t, \tau) G(\tau, s) h(s) d s d \tau:=\operatorname{Sh}(t)
$$

If $h \in C(I)$, the solution is in $C^{4}(I)$ and is a classical solution. Moreover, the solution operator of $\operatorname{LBVP}(10), S: L^{2}(I) \rightarrow$ $W^{4,2}(I)$ is a linearly bounded operator. By the compactness of the Sobolev embedding $W^{4,2}(I) \hookrightarrow C^{3}(I)$ and the continuity of embedding $C^{3}(I) \hookrightarrow W^{3,2}(I)$, we see that $S$ maps $L^{2}(I)$ into $W^{3,2}(I)$ and $S: L^{2}(I) \rightarrow W^{3,2}(I)$ is a completely continuous operator.

Choose a subspace of $W^{3,2}(I)$ by

$$
D=\left\{u \in W^{3,2}(I) \mid u(0)=u(1)=0, u^{\prime \prime}(0)=u^{\prime \prime}(1)=0\right\} .
$$

Clearly, $D$ is a closed subspace, and hence $D$ is a Banach space by the norm $\|u\|_{3,2}$ of $W^{3,2}(I)$. Define another norm on $D$ by

$$
\|u\|_{X}=\left\|u^{\prime \prime \prime}\right\|_{2}, \quad u \in D .
$$

One easily verifies that $\|u\|_{X}$ is equivalent to $\|u\|_{3,2}$. Hereafter, we use $X$ to denote the Banach space $D$ endowed the norm $\|u\|_{X}$, namely,

$$
X=\left(D,\|\cdot\|_{X}\right) .
$$

By the boundary condition of $\operatorname{LBVP}(10)$, the solution operator $S$ maps $H$ into $D$. Hence $S: H \rightarrow X$ is completely continuous.

Lemma 4. For $\operatorname{LBVP}(10)$, the following two conclusions hold.

(a) The norm of the solution operator of $L B V P(10) S$ : $H \rightarrow X$ satisfies $\|S\|_{\mathscr{L}(H, X)} \leq 1 / \pi$. 
(b) For every $h \in H$, the unique solution of $L B V P(10) u \in$ $W^{4,2}(I)$ satisfies the inequalities

$$
\begin{aligned}
& \|u\|_{2} \leq \frac{1}{\pi^{3}}\left\|u^{\prime \prime \prime}\right\|_{2}, \\
& \left\|u^{\prime}\right\|_{2} \leq \frac{1}{\pi^{2}}\left\|u^{\prime \prime \prime}\right\|_{2}, \\
& \left\|u^{\prime \prime}\right\|_{2} \leq \frac{1}{\pi}\left\|u^{\prime \prime \prime}\right\|_{2} .
\end{aligned}
$$

Proof. Since sine system $\{\sin k \pi t \mid k \in \mathbb{N}\}$ is a complete orthogonal system of $L^{2}(I)$, every $h \in L^{2}(I)$ can be expressed by the Fourier series expansion

$$
h(t)=\sum_{k=1}^{\infty} h_{k} \sin k \pi t,
$$

where $h_{k}=2 \int_{0}^{1} h(s) \sin k \pi s d s, k=1,2, \ldots$, and the Parseval equality

$$
\|h\|_{2}^{2}=\frac{1}{2} \sum_{k=1}^{\infty}\left|h_{k}\right|^{2},
$$

holds. Let $u=S h$; then $u \in W^{4,2}(I)$ is the unique solution of $\operatorname{LBVP}(10)$, and $u, u^{\prime \prime}$, and $u^{(4)}$ can be expressed by the Fourier series expansion of the sine system. Since $u^{(4)}=h$, by the integral formula of Fourier coefficient, we obtain that

$$
\begin{gathered}
u(t)=\sum_{k=1}^{\infty} \frac{h_{k}}{k^{4} \pi^{4}} \sin k \pi t, \\
u^{\prime \prime}(t)=-\sum_{k=1}^{\infty} \frac{h_{k}}{k^{2} \pi^{2}} \sin k \pi t .
\end{gathered}
$$

On the other hand, since cosine system $\{\cos k \pi t \mid k=$ $0,1,2, \ldots\}$ is another complete orthogonal system of $L^{2}(I)$, every $v \in L^{2}(I)$ can be expressed by the cosine series expansion

$$
v(t)=\frac{a_{0}}{2}+\sum_{k=1}^{\infty} a_{k} \cos k \pi t,
$$

where $a_{k}=2 \int_{0}^{1} h(s) \cos k \pi s d s, k=0,1,2, \ldots$. For the above $u=S h$, by the integral formula of the coefficient of cosine series, we obtain the cosine series expansions of $u^{\prime}$ and $u^{\prime \prime \prime}$ :

$$
\begin{aligned}
& u^{\prime}(t)=\sum_{k=1}^{\infty} \frac{h_{k}}{k^{3} \pi^{3}} \cos k \pi t, \\
& u^{\prime \prime \prime}(t)=-\sum_{k=1}^{\infty} \frac{h_{k}}{k \pi} \cos k \pi t .
\end{aligned}
$$

Now from (23), (19), and Parseval equality, it follows that

$$
\begin{aligned}
\|S h\|_{X}^{2} & =\left\|u^{\prime \prime \prime}\right\|_{2}^{2}=\frac{1}{2} \sum_{k=1}^{\infty}\left|\frac{h_{k}}{k \pi}\right|^{2} \\
& \leq \frac{1}{2 \pi^{2}} \sum_{k=-\infty}^{\infty}\left|h_{k}\right|^{2}=\frac{1}{\pi^{2}}\|h\|_{2}^{2} .
\end{aligned}
$$

This means that $\|S\|_{\mathscr{L}(H, X)} \leq 1 / \pi$, namely, (a) holds.
By (20)-(22) and Paserval equality, we have that

$$
\begin{aligned}
\|u\|_{2}^{2} & =\frac{1}{2} \sum_{k=1}^{\infty}\left|\frac{h_{k}}{k^{4} \pi^{4}}\right|^{2} \\
& \leq \frac{1}{2 \pi^{6}} \sum_{k=1}^{\infty}\left|\frac{h_{k}}{k \pi}\right|^{2}=\frac{1}{\pi^{6}}\left\|u^{\prime \prime \prime}\right\|_{2}^{2}, \\
\left\|u^{\prime}\right\|_{2}^{2} & =\frac{1}{2} \sum_{k=1}^{\infty}\left|\frac{h_{k}}{k^{3} \pi^{3}}\right|^{2} \\
& \leq \frac{1}{2 \pi^{4}} \sum_{k=1}^{\infty}\left|\frac{h_{k}}{k \pi}\right|^{2}=\frac{1}{\pi^{4}}\left\|u^{\prime \prime \prime}\right\|_{2}^{2}, \\
\left\|u^{\prime \prime}\right\|_{2}^{2} & =\frac{1}{2} \sum_{k=1}^{\infty}\left|\frac{h_{k}}{k^{2} \pi^{2}}\right|^{2} \\
& \leq \frac{1}{2 \pi^{2}} \sum_{k=1}^{\infty}\left|\frac{h_{k}}{k \pi}\right|^{2}=\frac{1}{\pi^{2}}\left\|u^{\prime \prime \prime}\right\|_{2}^{2} .
\end{aligned}
$$

This shows that the conclusion (b) holds.

Proof of Theorem 1. We define a mapping $F: X \rightarrow H$ by

$$
F(u)(t):=f\left(t, u(t), u^{\prime}(t), u^{\prime \prime}(t), u^{\prime \prime \prime}(t)\right), \quad u \in X .
$$

From the assumption (6) and the property of Carathéodory mapping it follows that $F: X \rightarrow H$ is continuous and it maps every bounded set of $X$ into a bounded set of $H$. Hence, the composite mapping $S \circ F: X \rightarrow X$ is completely continuous. We use the Leray-Schauder fixed-point theorem to show that $S \circ F$ has at least one fixed-point. For this, we consider the homotopic family of the operator equations:

$$
u=\lambda(S \circ F)(u), \quad 0<\lambda<1 .
$$

We need to prove that the set of the solutions of (29) is bounded in $X$. See [14].

Let $u \in X$ be a solution of an equation of (29) for $\lambda \in(0,1)$. Set $h=\lambda F(u)$; then by the definition of $S, u=$ Sh $\in W^{4,2}(I)$ is the unique solution of $\operatorname{LBVP}(10)$. By (a) of Lemma 4, we have

$$
\begin{aligned}
\|u\|_{X} & =\|S h\|_{X} \leq\|S\|_{\mathscr{L}(X, H)}\|h\|_{2} \\
& \leq \frac{1}{\pi}\|h\|_{2} \leq \frac{1}{\pi}\|F(u)\|_{2} .
\end{aligned}
$$

From (28), (6), and (b) of Lemma 4, it follows that

$$
\begin{aligned}
\|F(u)\|_{2} & \leq c_{0}\|u\|_{2}+c_{1}\left\|u^{\prime}\right\|_{2}+c_{2}\left\|u^{\prime \prime}\right\|_{2}+c_{3}\left\|u^{\prime \prime \prime}\right\|_{2}+M \\
& \leq\left(\frac{c_{0}}{\pi^{3}}+\frac{c_{1}}{\pi^{2}}+\frac{c_{2}}{\pi}+c_{3}\right)\left\|u^{\prime \prime \prime}\right\|_{2}+M \\
& =\pi\left(\frac{c_{0}}{\pi^{4}}+\frac{c_{1}}{\pi^{3}}+\frac{c_{2}}{\pi^{2}}+\frac{c_{3}}{\pi}\right)\|u\|_{X}+M .
\end{aligned}
$$


Combining this inequality with (30), we obtain that

$$
\|u\|_{X} \leq \frac{M}{1-\left(c_{0} / \pi^{4}+c_{1} / \pi^{3}+c_{2} / \pi^{2}+c_{3} / \pi\right)}:=C_{0} .
$$

This means that the set of the solutions for (29) is bounded in $X$. Therefore, by the Leray-Schauder fixed-point theorem [14], $S \circ F$ has a fixed-point $u_{0} \in X$. Let $h_{0}=F\left(u_{0}\right)$. By the definition of $S, u_{0}=S h_{0} \in W^{4,2}(I)$ is a solution of $\operatorname{LBVP}(10)$ for $h=h_{0}$. Since $W^{4,2}(I) \hookrightarrow C^{3}(I)$, from (28) it follows that $h_{0} \in C(I)$. Hence $u_{0} \in C^{4}(I)$ is a classical solution of $\operatorname{LBVP}(10)$, and by (28) $u_{0}$ is also a solution of $\operatorname{BVP}(1)$.

The proof of Theorem 1 is completed.

Proof of Theorem 2. Let $M=\max \{|f(t, 0,0,0,0)|: t \in I\}+1$. From condition (8) of Theorem 2 we easily see that Condition (6) of Theorem 1 holds. By Theorem 1, the BVP(1) has at least one solution.

Now, let $u_{1}, u_{2} \in C^{4}(I)$ be two solutions of $\operatorname{BVP}(1)$; then $u_{i}=S\left(F\left(u_{i}\right)\right), i=1,2$. From (8) and (28), we obtain that

$$
\left|F\left(u_{2}\right)(t)-F\left(u_{1}\right)(t)\right| \leq+\sum_{i=0}^{3} c_{i}\left|u_{2}^{(i)}(t)-u_{1}^{(i)}(t)\right|,
$$

for $t \in I$. Since $u_{2}-u_{1}$ is the solution of $\operatorname{LBVP}(10)$ for $h=$ $F\left(u_{2}\right)-F\left(u_{1}\right)$, by (33) and (b) of Lemma 4 , we have

$$
\begin{aligned}
\left\|F\left(u_{2}\right)-F\left(u_{1}\right)\right\|_{2} & \leq \sum_{i=0}^{3} c_{i}\left\|u_{2}^{(i)}-u_{1}^{(i)}\right\|_{2} \\
& \leq\left(\frac{c_{0}}{\pi^{3}}+\frac{c_{1}}{\pi^{2}}+\frac{c_{2}}{\pi}+c_{3}\right)\left\|u_{2}^{\prime \prime \prime}-u_{1}^{\prime \prime \prime}\right\|_{2} \\
& =\pi\left(\frac{c_{0}}{\pi^{4}}+\frac{c_{1}}{\pi^{3}}+\frac{c_{2}}{\pi^{2}}+\frac{c_{3}}{\pi}\right)\left\|u_{2}-u_{1}\right\|_{X} .
\end{aligned}
$$

From this and (a) of Lemma 4, it follows that

$$
\begin{aligned}
\left\|u_{2}-u_{1}\right\|_{X} & =\left\|S\left(F\left(u_{2}\right)-F\left(u_{1}\right)\right)\right\|_{X} \\
& \leq\|S\|_{\mathscr{L}(X, H)}\left\|F\left(u_{2}\right)-F\left(u_{1}\right)\right\|_{2} \\
& \leq\left(\frac{c_{0}}{\pi^{4}}+\frac{c_{1}}{\pi^{3}}+\frac{c_{2}}{\pi^{2}}+\frac{c_{3}}{\pi}\right)\left\|u_{2}-u_{1}\right\|_{X} .
\end{aligned}
$$

Since $c_{0} / \pi^{4}+c_{1} / \pi^{3}+c_{2} / \pi^{2}+c_{3} / \pi<1$, from (35) we see that $\left\|u_{2}-u_{1}\right\|_{X}=0$, that is $u_{2}=u_{1}$. Therefore, BVP(1) has only one solution.

The proof of Theorem 2 is completed.

Example 5. Consider the following fully linear fourth-order boundary value problem

$$
\begin{gathered}
u^{(4)}(t)=a_{0}(t) u(t)+a_{1}(t) u^{\prime}(t)+a_{2}(t) u^{\prime \prime}(t) \\
+a_{3}(t) u^{\prime \prime \prime}(t)+h(t), \quad t \in I, \\
u(0)=u(1)=u^{\prime \prime}(0)=u^{\prime \prime}(1)=0,
\end{gathered}
$$

where the coefficient functions $a_{0}, a_{1}, a_{2}, a_{3} \in C(I)$ and the inhomogeneous term $h \in C(I)$. All the known results of [113] are not applicable to this equation. Let

$$
\begin{gathered}
f\left(t, x_{0}, x_{1}, x_{2}, x_{3}\right)=a_{0}(t) x_{0}+a_{1}(t) x_{1} \\
+a_{2}(t) x_{2}+a_{3}(t) x_{3}+h(t), \\
c_{i}=\max _{t \in I}\left|a_{i}(t)\right|, \quad i=0,1,2,3 .
\end{gathered}
$$

It is easy to see that the partial derivatives $f_{x_{0}}, f_{x_{1}}, f_{x_{2}}$, and $f_{x_{3}}$ exist and

$$
\left|f_{x_{i}}\left(t, x_{0}, x_{1}, x_{2}, x_{3}\right)\right|=\left|a_{i}(t)\right| \leq c_{i}, \quad i=0,1,2,3 .
$$

Assume that the constants $c_{0}, c_{1}, c_{2}, c_{3}$ satisfy (7). Then by Corollary $3,(36)$ has a unique solution.

Example 6. Consider the following nonlinear fourth-order boundary value problem

$$
\begin{gathered}
u^{(4)}(t)=\sum_{i=0}^{3} b_{i}(t)\left|u^{(i)}(t)\right|^{\alpha_{i}}+\sin \pi t, \quad t \in I, \\
u(0)=u(1)=u^{\prime \prime}(0)=u^{\prime \prime}(1)=0
\end{gathered}
$$

where $b_{i} \in C(I), \alpha_{i} \in(0,1), i=0,1,2,3$. Let

$$
f\left(t, x_{0}, x_{1}, x_{2}, x_{3}\right)=\sum_{i=0}^{3} b_{i}(t)\left|x_{i}\right|^{\alpha_{i}}+\sin \pi t .
$$

Then $f \in C\left([0,1] \times \mathbb{R}^{4}, \mathbb{R}\right)$ and it satisfies that

$$
\lim _{\left|x_{0}\right|+\left|x_{1}\right|+\left|x_{2}\right|+\left|x_{3}\right| \rightarrow \infty} \max _{t \in I} \frac{f\left(t, x_{0}, x_{1}, x_{2}, x_{3}\right)}{\left|x_{0}\right|+\left|x_{1}\right|+\left|x_{2}\right|+\left|x_{3}\right|}=0 .
$$

From this one easily proves that there exists a positive constant $M>0$ such that

$$
\left|f\left(t, x_{0}, x_{1}, x_{2}, x_{3}\right)\right| \leq\left|x_{0}\right|+\left|x_{1}\right|+\left|x_{2}\right|+\left|x_{3}\right|+M .
$$

Since (7) holds for the constants $c_{0}=c_{1}=c_{2}=c_{3}=1$, by (42) $f$ satisfies the conditions of Theorem 1 . Hence by Theorem 1, (39) has at least one solution. This conclusion cannot be obtained from the results in [1-13].

\section{Acknowledgments}

This research is supported by NNSFs of China (11261053, $11061031)$ and the NFS of Gansu province (1208RJZA129).

\section{References}

[1] A. R. Aftabizadeh, "Existence and uniqueness theorems for fourth-order boundary value problems," Journal of Mathematical Analysis and Applications, vol. 116, no. 2, pp. 415-426, 1986.

[2] Y. S. Yang, "Fourth-order two-point boundary value problems," Proceedings of the American Mathematical Society, vol. 104, no. 1, pp. 175-180, 1988. 
[3] M. A. del Pino and R. F. Manásevich, "Existence for a fourthorder boundary value problem under a two-parameter nonresonance condition," Proceedings of the American Mathematical Society, vol. 112, no. 1, pp. 81-86, 1991.

[4] C. De Coster, C. Fabry, and F. Munyamarere, "Nonresonance conditions for fourth order nonlinear boundary value problems," International Journal of Mathematics and Mathematical Sciences, vol. 17, no. 4, pp. 725-740, 1994.

[5] M. Ruyun, Z. Jihui, and F. Shengmao, "The method of lower and upper solutions for fourth-order two-point boundary value problems," Journal of Mathematical Analysis and Applications, vol. 215, no. 2, pp. 415-422, 1997.

[6] Z. Bai, "The method of lower and upper solutions for a bending of an elastic beam equation," Journal of Mathematical Analysis and Applications, vol. 248, no. 1, pp. 195-202, 2000.

[7] Y. Li, "Two-parameter nonresonance condition for the existence of fourth-order boundary value problems," Journal of Mathematical Analysis and Applications, vol. 308, no. 1, pp. 121-128, 2005.

[8] Y. Li, "On the existence of positive solutions for the bending elastic beam equations," Applied Mathematics and Computation, vol. 189, no. 1, pp. 821-827, 2007.

[9] C. P. Gupta, "Existence and uniqueness results for the bending of an elastic beam equation at resonance," Journal of Mathematical Analysis and Applications, vol. 135, no. 1, pp. 208-225, 1988.

[10] R. P. Agarwal, "On fourth order boundary value problems arising in beam analysis," Differential and Integral Equations, vol. 2, no. 1, pp. 91-110, 1989.

[11] Z. Bai and H. Wang, "On positive solutions of some nonlinear fourth-order beam equations," Journal of Mathematical Analysis and Applications, vol. 270, no. 2, pp. 357-368, 2002.

[12] F. Li, Q. Zhang, and Z. Liang, "Existence and multiplicity of solutions of a kind of fourth-order boundary value problem," Nonlinear Analysis. Theory, Methods \& Applications A, vol. 62, no. 5, pp. 803-816, 2005.

[13] G. Han and F. Li, "Multiple solutions of some fourth-order boundary value problems," Nonlinear Analysis. Theory, Methods \& Applications A, vol. 66, no. 11, pp. 2591-2603, 2007.

[14] K. Deimling, Nonlinear Functional Analysis, Springer, New York, NY, USA, 1985. 


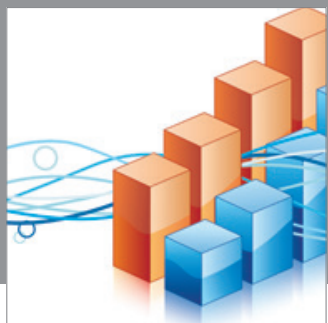

Advances in

Operations Research

mansans

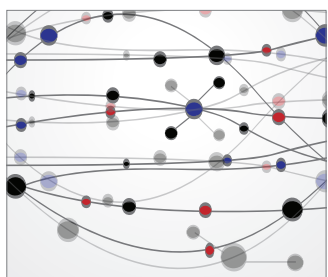

The Scientific World Journal
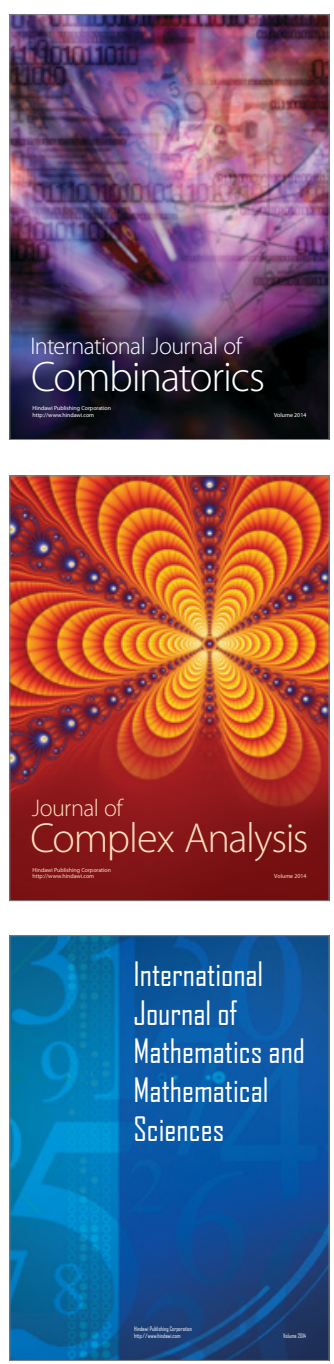
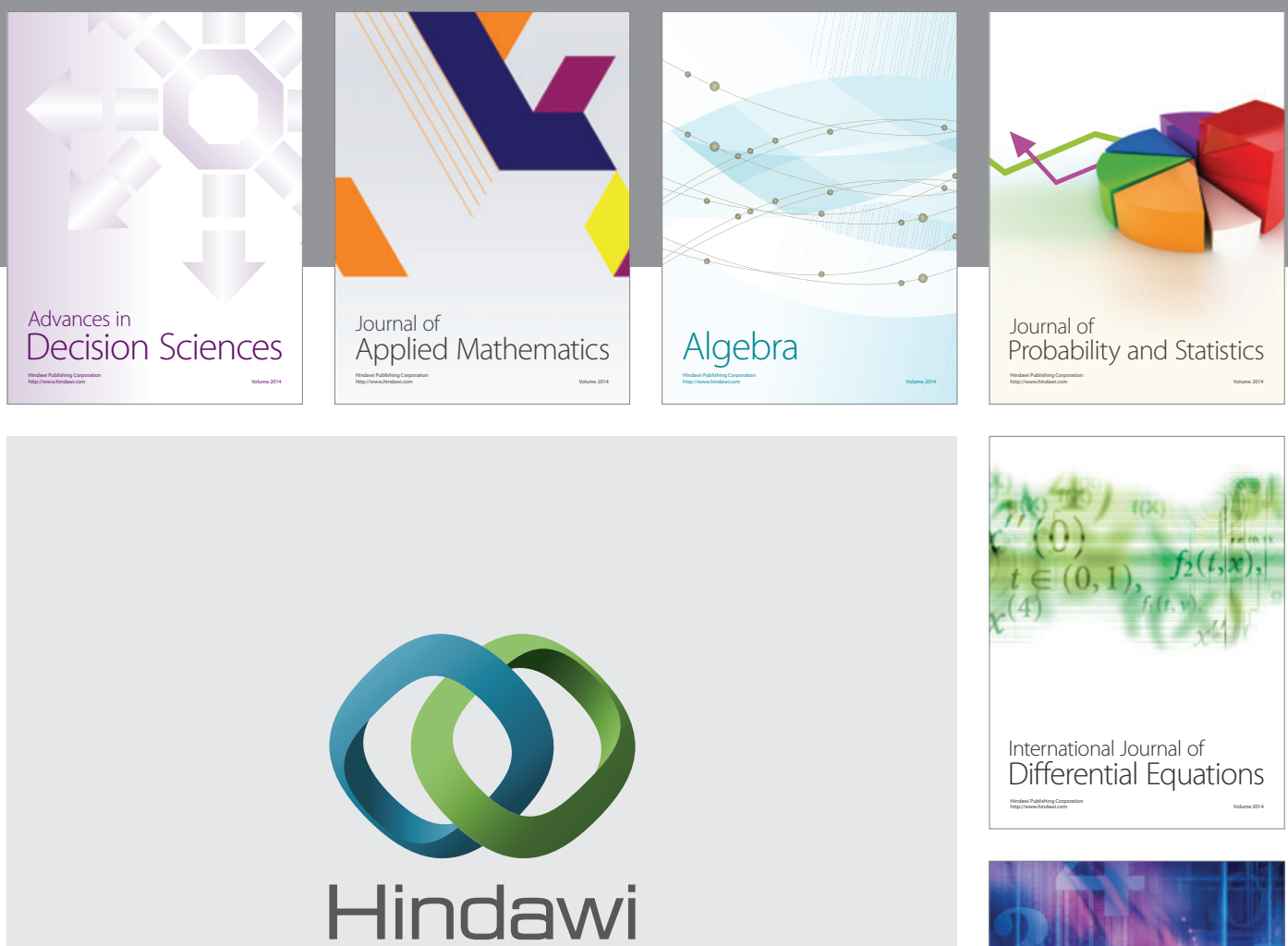

Submit your manuscripts at http://www.hindawi.com
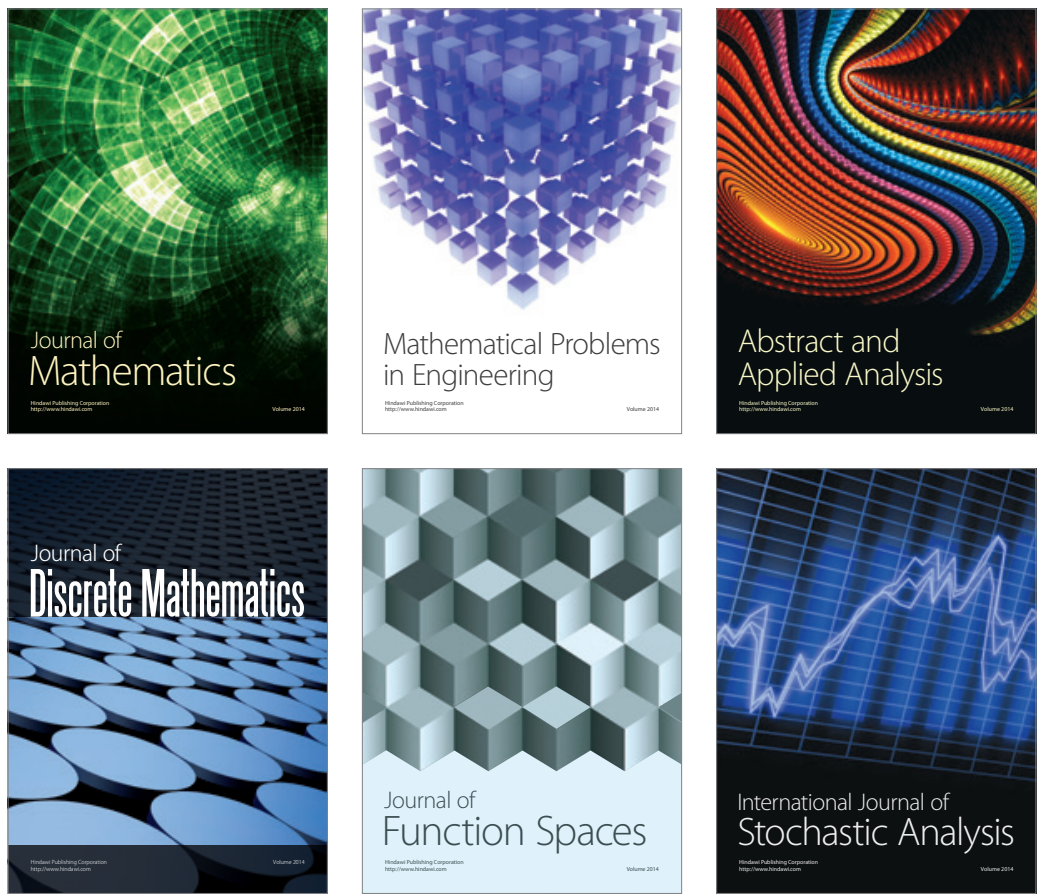

Journal of

Function Spaces

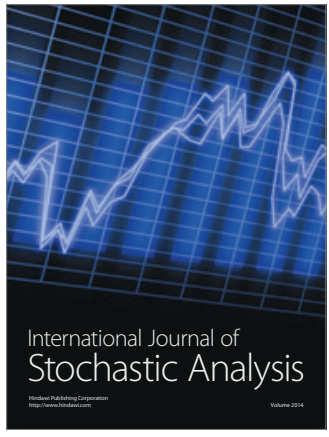

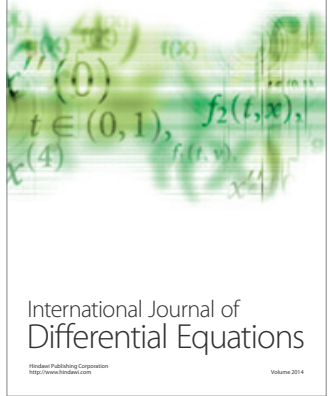
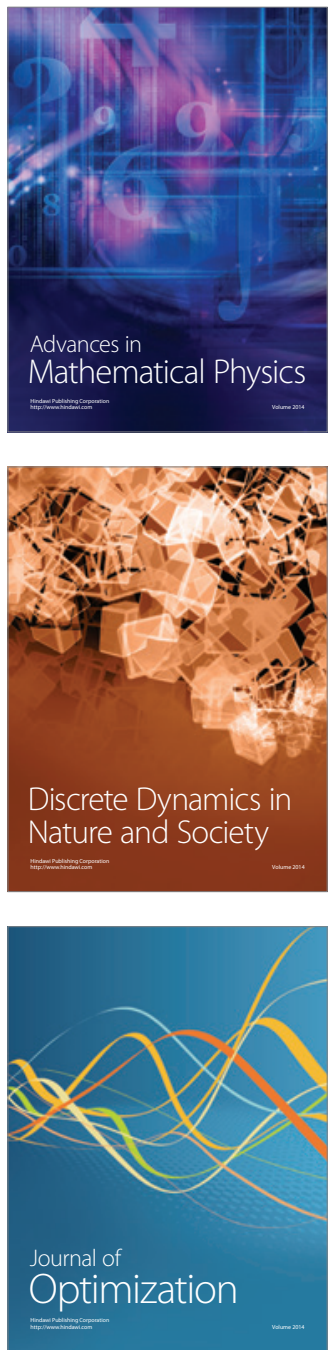